\title{
Optical characterization and reverse engineering based on multiangle spectroscopy
}

\author{
Alexander V. Tikhonravov,${ }^{1, *}$ Tatiana V. Amotchkina, ${ }^{1}$ Michael K. Trubetskov, ${ }^{1}$ \\ Robert J. Francis, ${ }^{2}$ Vesna Janicki, ${ }^{3}$ Jordi Sancho-Parramon, ${ }^{3}$ \\ Hrvoje Zorc, ${ }^{3}$ and Vladimir Pervak ${ }^{4}$ \\ ${ }^{1}$ Research Computing Center, Moscow State University, Leninskie Gory, 119992, Moscow, Russia \\ ${ }^{2}$ Agilent Technologies, 679 Springvale Road, Mulgrave, Victoria 3170, Australia \\ ${ }^{3}$ Rudjer Boskovic Institute, Bijenicka cesta 54, 10002, Zagreb, Croatia \\ ${ }^{4}$ Ludwig-Maximilians-Universität München, Am Coulombwall 1, D-85748 Garching, Germany \\ ${ }^{*}$ Corresponding author: tikh@srcc.msu.ru
}

Received 15 July 2011; revised 5 October 2011; accepted 6 October 2011; posted 7 October 2011 (Doc. ID 151125); published 9 January 2012

\begin{abstract}
We perform characterization of thin films and reverse engineering of multilayer coatings on the basis of multiangle spectral photometric data provided by a new advanced spectrophotometer accessory. Experimental samples of single thin films and multilayer coatings are produced by magnetron sputtering and electron-beam evaporation. Reflectance and transmittance data at two polarization states are measured at incidence angles from 7 to $40 \mathrm{deg}$. We demonstrate that multiangle reflectance and transmittance data provide reliable characterization and reverse-engineering results. (C) 2012 Optical Society of America OCIS codes: $\quad 310.3840,310.6860$.
\end{abstract}

\section{Introduction}

Determination of optical parameters for thin films (optical characterization of thin films) is practically important because successful manufacturing of highquality coatings is directly dependent upon the accuracy of knowing the optical parameters of thin films. Therefore, it is not surprising that this problem was one of the focuses of the three last Topical Meetings on Optical Interference Coatings [1-ㅗ].

Reliable estimation of thicknesses and optical parameters of layers of produced multilayer coatings (reverse engineering of optical coatings) is also of great importance because it provides a feedback to the design-production chain. Reverse-engineering results can be used for adjusting deposition parameters, recalibrating monitoring systems, and improving control of thicknesses of individual layers.

$1559-128 \mathrm{X} / 12 / 020245-10 \$ 15.00 / 0$

(C) 2012 Optical Society of America
Typically, optical characterization is based on the analysis of normal- or near-normal-incidence transmittance $(T)$ and/or reflectance $(R)$ data of a thin film sample on a transparent substrate. This characterization approach is the most popular one because it is simple from an experimental point of view. Transmittance at normal incidence can be measured with a sufficient accuracy on any UV-visible-near-IR (UV-VIS-NIR) or Fourier-transform-IR spectrophotometer without the need for a polarizer or special accessory. Near-normal reflectance can be also measured on most of these instruments with a nonpolarizing accessory.

Optical characterization based on normalincidence $R$ and $T$ measurements requires applying a special methodology for the reliable determination of thin film optical parameters [4,5]. In comparison with optical characterization of single thin films, reverse engineering of multilayer stacks is an even more complicated problem because, in general, more unknown parameters are to be found. Specific mathematical algorithms are required for solving various 
reverse-engineering problems [6-8]. Reliable reverse engineering based on normal-incidence or nearnormal-incidence $T$ and $R$ measurement data is a challenging problem. To find layer parameters reliably, it is required to properly apply a sequence of physically sensible low-parametric models of optical constants and thickness errors [9]. The main problem with a great number of unknown parameters is a possible nonuniqueness of their determination. The ambiguity of reverse engineering grows with a growing number of coating layers. The ambiguity of optical characterization based on normal- or nearnormal-incidence $R$ and $T$ measurement data is also a big problem when stacked thin films or absorbing films are considered [10-12].

A general approach to overcoming possible ambiguity in reverse engineering and optical characterization is based on utilizing more measurement data. In particular, not only normal-incidence but also oblique-incidence $R$ and $T$ data can be used for thin film characterization [11,13-17]. In this paper, we perform a systematic study of the applicability of multiangle spectral photometric data to the optical characterization of single thin films and the reverse engineering of multilayer optical coatings. We consider dense thin films and a multilayer produced by magnetron sputtering, as well as electron-beam (e-beam) evaporated thin films, for which characterization is typically more difficult.

In Section 2, we describe the new measurement device and two sets of samples produced by different deposition techniques. We use these samples for the systematic study of the applicability of multiangle spectroscopy to optical characterization of single thin films and reverse engineering of multilayer coatings. In Section 3, we use single-layer samples produced by magnetron sputtering to check the consistency of characterization results obtained from spectral photometric data measured at different incidence angles. In Section 4 , we consider a multilayer sample produced by magnetron sputtering and use this sample to demonstrate reliability of reverseengineering results obtained using multiangle spectral photometric data. In Section 5 , we propose a methodology of using multiangle spectroscopy for optical characterization of e-beam evaporated films with essentially different thicknesses. Conclusions on our research are presented in Section $\underline{6}$.

\section{Experimental Samples and Measurement Process}

For our study we produced two sets of experimental samples using two different deposition techniques: magnetron sputtering and e-beam evaporation. A short description of these samples is presented in Table 1 . The main reason for choosing two different deposition techniques is as follows. Magnetron sputtering is known as a process with a stable deposition rate and very dense layers (see, for example, [18,19]). In this situation, one has assurance in the stability and amorphous structure of produced films, which facilitates optical characterization and reverse engineering.

The first set of experimental samples was produced with magnetron sputtering in the Leybold Optics Helios plant. This plant is equipped with two proprietary TwinMags magnetrons and a plasma source for plasma/ion-assisted reactive middle frequency dual magnetron sputtering. The system was pumped by turbomolecular pumps to 1 . $10^{-6}$ mbar before the deposition. Argon and oxygen were used for both magnetrons. The cathode power for Si and Ta targets was $4500 \mathrm{~W}$ and $2500 \mathrm{~W}$, respectively. The power applied to the Ta cathode was not constant because it operated in the oxygen control mode, which guaranteed stable film properties. The gas pressure was $1 \cdot 10^{-3}$ mbar during the sputtering process. Oxygen was fed near the targets to oxidize the sputtering films. The distance from the targets to the substrates was $100 \mathrm{~mm}$. The chosen targets were $\mathrm{Si}$ and $\mathrm{Ta}$, and the purity of the $\mathrm{Si}$ and Ta targets was $99.999 \%$ and $99.8 \%$, respectively. The deposition rates were approximately $0.5 \mathrm{~nm} / \mathrm{s}$ for both materials.

The second set of samples was prepared by e-beam evaporation in a modified Varian 3117 chamber. The BK7 substrates were positioned onto a rotating calotte to ensure uniform thickness of the layers over the samples surface. Base pressure was $4 \cdot 10^{-6}$ Torr. Partial pressure of oxygen during deposition of silica was $5 \cdot 10^{-5}$ Torr and during deposition of $\mathrm{HfO}_{2}$, it was $9 \cdot 10^{-5}$ Torr. The layer mass thickness was

Table 1. Description of Experimental Samples

\begin{tabular}{lcc}
\hline Sample Structure & Substrate Material/Thickness & Deposition Technique \\
\hline Uncoated substrate & BK7/1.00 mm & magnetron sputtering \\
Single layer of $\mathrm{SiO}_{2}$ & Suprasil/6.35 mm & magnetron sputtering \\
Single layer of $\mathrm{Ta}_{2} \mathrm{O}_{5}$ & Suprasil/6.35 mm & magnetron sputtering \\
15 -layer QWM with central wavelength $600 \mathrm{~nm}$ & Suprasil/6.35 mm & \\
$\left(\right.$ odd layers, $\mathrm{Ta}_{2} \mathrm{O}_{5} ;$ even layers, $\left.\mathrm{SiO}_{2}\right)$ & & \\
Single layer of $\mathrm{HfO}_{2}$ & BK7/1 mm & e-beam evaporation \\
Single layer of $\mathrm{SiO}_{2}$ & BK7/1 mm & e-beam evaporation \\
12 -layer $\mathrm{QWM} \mathrm{with} \mathrm{central} \mathrm{wavelength} 410 \mathrm{~nm}$ & BK7/1 mm & e-beam evaporation \\
(odd layers, $\mathrm{HfO}_{2}$; even layers, $\mathrm{SiO}_{2}$ ) & & \\
\hline
\end{tabular}

QWM: quarter-wave mirror; see Section 4 . 
controlled by a quartz crystal monitor. Deposition rates were $10 \AA / \mathrm{s}$ for $\mathrm{SiO}_{2}$ and 10-15 $\mathrm{A} / \mathrm{s}$ for $\mathrm{HfO}_{2}$.

We used samples produced by magnetron sputtering for demonstrating the reliability of both procedures based on multiangle spectroscopy. In the case of samples produced by e-beam evaporation, it may be required to take into account thin film bulk inhomogeneity (variation of density over the depth of the film) [4] and other structure properties, which makes optical characterization and reverse engineering more difficult. In our study we applied multiangle spectroscopy for the determination of optical parameters of e-beam evaporated thin films with essentially different thicknesses which, as we show, affects their optical parameters.

For all samples, multiangle spectral photometric measurements were performed using recently developed advanced accessory to the double beam UV-VIS-NIR-type spectrophotometer Cary 5000 developed by Agilent Technologies. The schematic of the accessory is presented in Fig. 1. In the absolute variable angle reflectance and transmittance accessory, the linearly polarized beam that illuminates the sample can be measured in transmission or, by moving the detector assembly in a plane at a constant radius from the sample, in reflectance. The baseline $(100 \%)$ for each polarization direction was measured in the transmittance detector position in the absence of a sample. The range of measurement angles was limited by the sample width $(25 \mathrm{~mm})$ and thickness $(6.35 \mathrm{~mm})$, since both the main transmitted beam and at least the first double internal reflection (in transmission) or main beam and first reflection from the back face (in reflection) needed to be unobstructed and included in the measurement. Most measurements were made with a beam convergence of $\pm 3^{\circ}$ horizontally and vertically, and with a spectral bandwidth of $4 \mathrm{~nm}$, but some were made with beam convergences of $\pm 2^{\circ}$, or with a bandwidth of $2 \mathrm{~nm}$, to check for sensitivity to these parameters. The geometry of this accessory is similar to that presented in [20]: the sample and the detector assembly rotate about a common axis, and the reference or baseline position of the detector is in the

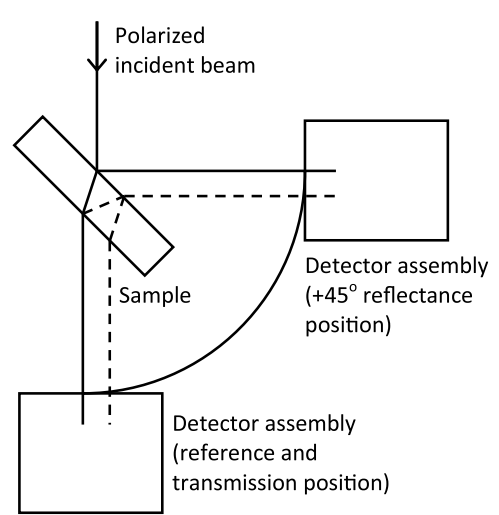

Fig. 1. Schematic of the absolute variable angle reflectance and transmission accessory. straight-through or $180^{\circ}$ position. At the same time, the polarizer and detector assemblies are quite different. The detector assembly has the uniformity and large aperture of an integrating sphere but without the bulk and the losses, making data collection faster.

Multiangle spectral photometric measurement data were performed for all samples described in Table 1 in the spectral range from $3002500 \mathrm{~nm}$ at incidence angles of $7^{\circ}, 10^{\circ}, 20^{\circ}, 30^{\circ}$, and $40^{\circ}$ for $s$ and $p$-polarized light. In all optical characterization and reverse-engineering procedures throughout this paper, we used only subsets of measurement data, namely, measurement data taken in the spectral range from 330 to $1100 \mathrm{~nm}$. Substrate internal absorption is significant above wavelength of $1100 \mathrm{~nm}$, making estimation of accuracy uncertain.

In [4] we discussed a methodology of optical characterization based on spectral photometric data. The first step of this methodology is estimating the accuracy of available measurement data. For this purpose, we use reflectance and transmittance data of the uncoated BK7 substrate with $1 \mathrm{~mm}$ thickness. In the spectral range from 400 to $1100 \mathrm{~nm}$, where absorption of BK7 glass is negligible, we estimate the measurement data error $\delta(\lambda)$ as

$$
\delta(\lambda)=100 \%-R(\lambda)-T(\lambda),
$$

where $R(\lambda)$ and $T(\lambda)$ are measured reflectance and transmittance data.

In the case of ideal measurements, $\delta(\lambda)$ should be equal to zero. In practice, $\delta(\lambda)$ differs from zero, and its average value in some spectral range can be considered as a level of error estimation within this range. We calculate $\delta(\lambda)$ values for arrays of measurement data corresponding to various incidence angles and polarization states. In Figs. $\underline{2}$ and $\underline{3}$ we show $\delta(\lambda)$ spectral dependencies corresponding to the $s$ - and $p$ polarization cases, respectively. One can see that the levels of error are different in the VIS and NIR spectral ranges. Changes in error level near 760 and $800 \mathrm{~nm}$ correspond to changes in spectrometer single beam signal level. The estimated levels of errors are

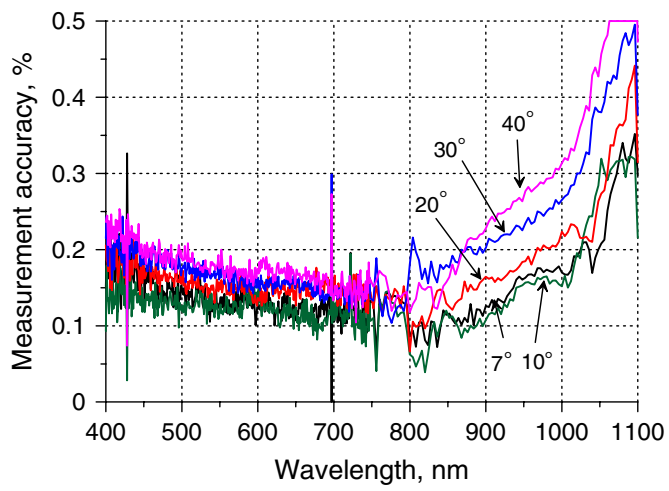

Fig. 2. (Color online) Estimating errors in measurement data in the $s$-polarization case: various spectral dependencies correspond to incidence angles of $7^{\circ}, 10^{\circ}, 20^{\circ}, 30^{\circ}$, and $40^{\circ}$. 


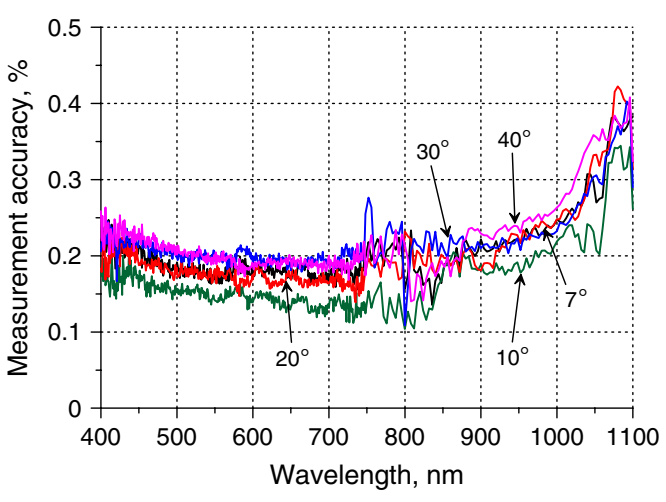

Fig. 3. (Color online) Estimating errors in measurement data in the $p$-polarization case: various spectral dependencies correspond to incidence angles of $7^{\circ}, 10^{\circ}, 20^{\circ}, 30^{\circ}$, and $40^{\circ}$.

presented in Table 2 . The level of errors in the VIS spectral range is lower than that in the NIR spectral range. It can be explained by lower sensitivity of the detector in the NIR spectral range. At the current state of the art in spectral photometric instrumentations, the levels of error presented in Table $\underline{2}$ can be considered as good ones $[4,21]$.

It is also important that, with the new multiangle accessory, levels of error are almost the same for all incidence angles and both polarization states. For optical characterization of thin films and reverse engineering of multilayer coatings, measurement data in the UV spectral range may also be important because $R$ and $T$ spectral dependencies typically have informative oscillating features in this range. For this reason, for our analysis we also use reflectance and transmittance data in the UV range starting from $330 \mathrm{~nm}$, where random noise in $R$ and $T$ data does not exceed $0.2 \%$.

\section{Optical Characterization of Dense Dielectric Thin Films Using Multiangle Spectral Photometric Data}

In principle, nonabsorbing or slightly absorbing dense dielectric thin films can be successfully characterized using normal- or near-normal-incidence spectral photometric data. A methodology for optical characterization of such thin films was discussed in [4]. In this section we use multiangle spectral

Table 2. Estimated Levels of Error in Measurement Data

\begin{tabular}{cccc}
\hline $\begin{array}{c}\text { Incidence } \\
\text { Angle }\end{array}$ & $\begin{array}{c}\text { Polarization } \\
\text { State }\end{array}$ & $\begin{array}{c}\text { Spectral } \\
\text { Range } \\
{[400 ; 800] \mathrm{nm}}\end{array}$ & $\begin{array}{c}\text { Spectral } \\
\text { Range } \\
{[800 ; 1100] \mathrm{nm}}\end{array}$ \\
\hline $7^{\circ}$ & $s$ & $0.10 \%$ & $0.16 \%$ \\
$10^{\circ}$ & $s$ & $0.10 \%$ & $0.16 \%$ \\
$20^{\circ}$ & $s$ & $0.13 \%$ & $0.19 \%$ \\
$30^{\circ}$ & $s$ & $0.14 \%$ & $0.24 \%$ \\
$40^{\circ}$ & $s$ & $0.15 \%$ & $0.27 \%$ \\
$7^{\circ}$ & $p$ & $0.15 \%$ & $0.23 \%$ \\
$10^{\circ}$ & $p$ & $0.12 \%$ & $0.19 \%$ \\
$20^{\circ}$ & $p$ & $0.15 \%$ & $0.23 \%$ \\
$30^{\circ}$ & $p$ & $0.17 \%$ & $0.24 \%$ \\
$40^{\circ}$ & $p$ & $0.17 \%$ & $0.24 \%$ \\
\hline
\end{tabular}

photometric data for optical characterization of $\mathrm{Ta}_{2} \mathrm{O}_{5}$ and $\mathrm{SiO}_{2}$ thin films produced by magnetron sputtering. This is mainly for demonstrating the consistency of the characterization results obtained from separate reflectance and transmittance measured at different incidence angles and at different polarization states. Results of optical characterization of $\mathrm{Ta}_{2} \mathrm{O}_{5}$ and $\mathrm{SiO}_{2}$ films are also required for reverse engineering of multilayer samples considered in Section 4 .

At our disposal we have ten separate files containing $R$ and $T$ data measured at different incidence angles with different polarization states. Characterization procedures exploring each separate data file follow the same steps according to the methodology described in [4].

At the first step, we calculate total losses in $\mathrm{Ta}_{2} \mathrm{O}_{5}$ and $\mathrm{SiO}_{2}$ thin film samples and find that, in all cases, total losses are negligible in the spectral range from 330 to $1100 \mathrm{~nm}$. This means that produced $\mathrm{Ta}_{2} \mathrm{O}_{5}$ and $\mathrm{SiO}_{2}$ films are nonabsorbing in this spectral range, which is in agreement with literature data [22].

At the second step, we compare $R$ and $T$ data with reflectance and transmittance of the uncoated $\mathrm{Su}$ prasil substrate. Such comparison allows one to check whether films are homogeneous or inhomogeneous. Deviations of reflectance and transmittance extrema from corresponding spectral characteristics of the uncoated substrate do not exceed $0.1 \%$. These deviations can be neglected because they are in the range of measurement data error and we conclude that $\mathrm{Ta}_{2} \mathrm{O}_{5}$ and $\mathrm{SiO}_{2}$ films are homogeneous. Based on the above conclusions, we choose for optical characterization a model of nonabsorbing and homogeneous thin film. We use Cauchy formulas to describe refractive indices $n(\lambda)$ of the $\mathrm{Ta}_{2} \mathrm{O}_{5}$ and $\mathrm{SiO}_{2}$ films. Refractive index wavelength dependencies and thicknesses of $\mathrm{Ta}_{2} \mathrm{O}_{5}$ and $\mathrm{SiO}_{2}$ films are obtained as a result of a minimization of discrepancy function estimating deviations of model reflectance and transmittance data from measured $R$ and $T$ data. OptiChar characterization software is used for this purpose [6].

Refractive index wavelength dependencies of $\mathrm{Ta}_{2} \mathrm{O}_{5}$ and $\mathrm{SiO}_{2}$ films found using various separate oblique-incidence $R$ and $T$ data are collected in Figs. $\underline{4}$ and $\underline{5}$. In these figures, an excellent agreement among obtained wavelength dependencies is observed.

In Table $\underline{3}$ we present numerical results of optical characterization: found film thicknesses and refractive index values at $\lambda=600 \mathrm{~nm}$. There is remarkable consistency among results obtained using $R$ and $T$ data measured at different incidence angles and with different polarization states. For both materials, deviations of thickness and refractive index values from mean values in all columns of Table $\underline{3}$ are lower than $0.1 \%$ !

Refractive index wavelength dependencies of $\mathrm{Ta}_{2} \mathrm{O}_{5}$ and $\mathrm{SiO}_{2}$ films are required for reverse 


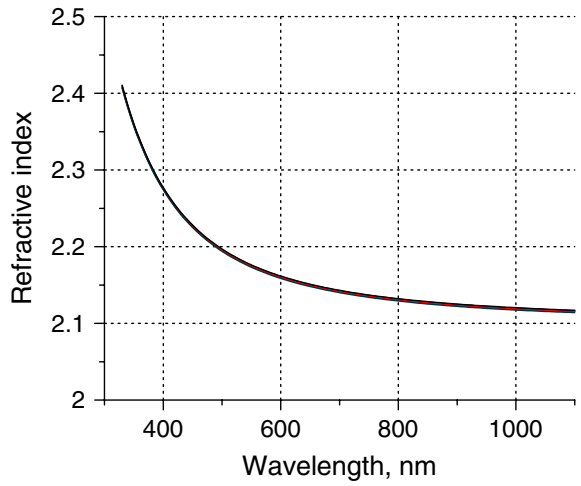

Fig. 4. Refractive indices of $\mathrm{Ta}_{2} \mathrm{O}_{5}$ found from separate obliqueincidence $R$ and $T$ measurements, for the $s$ - and $p$-polarization cases. The differences among the 10 presented wavelength dependencies are almost indistinguishable (see the text for detail).

engineering of multilayers based on these materials and will be used in Section $\underline{4}$. As final results of optical characterization of both thin film materials, we obtain nominal refractive indices as wavelength dependencies found on the basis of analysis of all available multiangle measurement data simultaneously (black curves in Fig. 6). These results are close to those obtained by averaging of wavelength dependencies depicted in Figs. $\underline{4}$ and $\underline{5}$.

\section{Reliability of Reverse Engineering Based on Multiangle Spectroscopy}

In this section, to check the reliability of reverse engineering based on multiangle optical photometric data, we use a specially prepared 15-layer quarterwave mirror with $\mathrm{Ta}_{2} \mathrm{O}_{5}$ and $\mathrm{SiO}_{2}$ as high and low index materials. The mirror was produced by magnetron sputtering by using time monitoring of layer thicknesses. During the deposition of this mirror, intentional errors of $+5 \%,-7 \%,-5 \%$, and $+5 \%$ were imposed on the third, eighth, 14th, and 15th mirror layers, respectively. These errors are several times higher than estimated levels of random errors associated with inaccuracies of the monitoring procedure. The level of absolute errors in layer thicknesses does not exceed 1-2 nm [18,23]. For our 15-layer quarter-

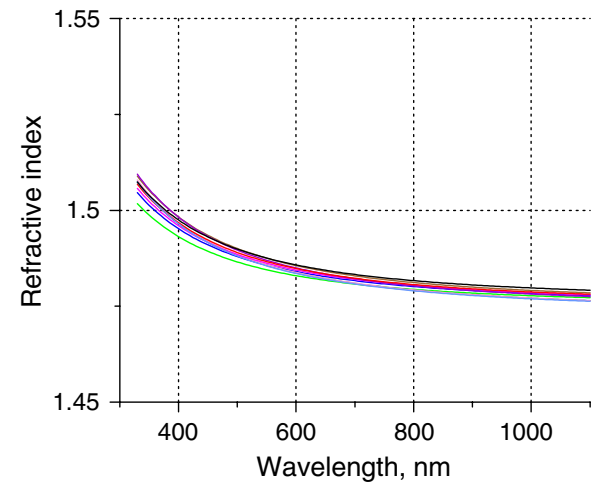

Fig. 5. (Color online) Refractive indices of $\mathrm{SiO}_{2}$ found using separate oblique-incidence $R$ and $T$ measurements, for the $s$ - and $p$-polarization cases (see the text for detail).
Table 3. Parameters of $\mathrm{Ta}_{2} \mathrm{O}_{5}$ and $\mathrm{SiO}_{2}$ Films Found by Using Separate Oblique-Incidence $R$ and $T$ Data

\begin{tabular}{|c|c|c|c|c|}
\hline \multirow[b]{2}{*}{$\begin{array}{c}\text { Polarization State/ } \\
\text { Angle } \\
\text { of Incidence }\end{array}$} & \multicolumn{2}{|c|}{$\mathrm{Ta}_{2} \mathrm{O}_{5}$} & \multicolumn{2}{|c|}{$\mathrm{SiO}_{2}$} \\
\hline & $\begin{array}{c}\text { Ph.th. }{ }^{a} \text {, } \\
\text { nm }\end{array}$ & $\begin{array}{c}n \text { at } \\
600 \mathrm{~nm}\end{array}$ & $\begin{array}{l}\text { Ph.th. }{ }^{\text {a }} \text { nm } \\
\text { nm }\end{array}$ & $\begin{array}{c}n \text { at } \\
600 \mathrm{~nm}\end{array}$ \\
\hline$s, 7^{\circ}$ & 292.3 & 2.162 & 401.4 & 1.486 \\
\hline$s, 10^{\circ}$ & 292.5 & 2.160 & 401.7 & 1.485 \\
\hline$s, 20^{\circ}$ & 292.4 & 2.161 & 401.5 & 1.484 \\
\hline$s, 30^{\circ}$ & 292.4 & 2.161 & 401.9 & 1.484 \\
\hline$s, 40^{\circ}$ & 292.4 & 2.161 & 401.6 & 1.483 \\
\hline$p, 7^{\circ}$ & 292.7 & 2.159 & 401.9 & 1.484 \\
\hline$p, 10^{\circ}$ & 292.5 & 2.160 & 401.4 & 1.485 \\
\hline$p, 20^{\circ}$ & 292.5 & 2.160 & 401.5 & 1.484 \\
\hline$p, 30^{\circ}$ & 292.5 & 2.160 & 401.9 & 1.486 \\
\hline$p, 40^{\circ}$ & 292.4 & 2.161 & 402.7 & 1.483 \\
\hline
\end{tabular}

${ }^{a}$ Ph.th.: physical thickness.

wave mirror, this corresponds to $1.4 \%-2.8 \%$ and $1 \%-2 \%$ relative accuracy in high and low refractive index layers, respectively.

We start reverse engineering of the produced mirror based on separate oblique-incidence $R$ and $T$ measurements. Figure 7 compares $R_{s}$ and $T_{s}$ measured at $7^{\circ}$ incidence and the corresponding theoretical characteristics of the 15-layer ideal quarterwave mirror without intended thickness errors. For calculating the theoretical characteristics, we use the nominal refractive indices of $\mathrm{Ta}_{2} \mathrm{O}_{5}$ and $\mathrm{SiO}_{2}$ found in Section 3.

In Fig. 7, one can see that measured characteristics are shifted to the shorter wavelength range with respect to theoretical characteristics. Analogous shifts are observed for all other measurement data files. A direct simulation of optical characteristics shows that these shifts cannot be connected with the intentional errors in layer thickness. Thus, the observed shifts are caused by deviations in actual layer optical thicknesses from theoretical optical thicknesses, which, in their turn, can be connected with the calibration procedure for time monitoring. In the case of the Helious plant, this calibration was performed based on so-called time-calibration refractive indices of $\mathrm{Ta}_{2} \mathrm{O}_{5}$ and $\mathrm{SiO}_{2}$ films presented in Fig. $\underline{6}$ by dashed curves. One can see that these
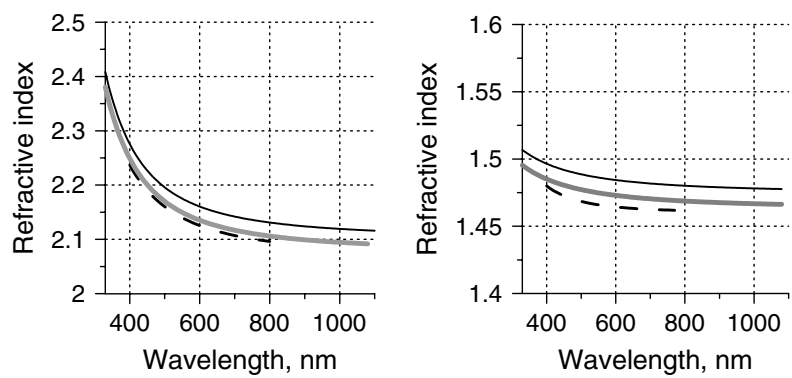

Fig. 6. Comparison of nominal refractive indices of $\mathrm{Ta}_{2} \mathrm{O}_{5}$ and $\mathrm{SiO}_{2}$ found from single layer measurements (solid black curves), corrected refractive indices found from multilayer data (gray curves), and time-calibration refractive indices (dashed curves). 

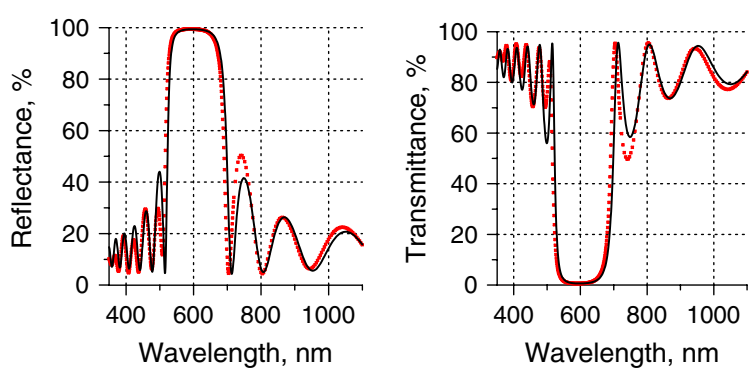

Fig. 7. (Color online) Initial fittings of measurement $R$ and $T$ data (crosses) by model reflectance and transmittance data (solid curves).

indices are somewhat lower than those used for calculating theoretical characteristics. We do not discuss here which results are more accurate. In principle, it is possible that the refractive indices of films in a multilayer stack are slightly different from the refractive indices of single thin films used for optical characterization because single films are several times thicker than films forming a quarter-wave stack. It is, however, important at the first step of reverse engineering to take into account factors that can be connected with calibration of the monitoring procedure. For this reason, we always start reverse engineering with accounting for possible offsets of refractive indices from the nominal ones presented in Fig. $\underline{6}$ by the black solid curves.

We determine refractive index offsets by minimizing the discrepancy function estimating a closeness between model and measured $R$ and $T$ data for any polarization:

$$
\begin{aligned}
\mathrm{DF}^{2}= & \frac{1}{L} \sum_{j=1}^{L}\left\{\left[R \left(n_{H}\left(\lambda_{j}\right)+h_{H}, n_{L}\left(\lambda_{j}\right)\right.\right.\right. \\
& \left.\left.+h_{L}, d_{1}, \ldots, d_{m}, \lambda_{j}, \theta\right)-\hat{R}\left(\lambda_{j}, \theta\right)\right]^{2}+ \\
& +\left[T \left(n_{H}\left(\lambda_{j}\right)+h_{H}, n_{L}(\lambda)\right.\right. \\
& \left.\left.\left.+h_{L}, d_{1}, \ldots, d_{m}, \lambda_{j}, \theta\right)-\hat{T}\left(\lambda_{j}, \theta\right)\right]^{2}\right\},
\end{aligned}
$$

where $d_{1}, \ldots, d_{m}$ are the theoretical thicknesses of coating layers, $L$ denotes the total number of points in the wavelength grid $\left\{\lambda_{j}\right\}$ in the considered spectral range, $\hat{R}$ and $\hat{T}$ denote measurement data, $n_{H}\left(\lambda_{j}\right)$ and $n_{L}\left(\lambda_{j}\right)$ are nominal high and low refractive indices, $h_{H}$ and $h_{L}$ are offsets of high and low refractive indices, respectively, and $\theta$ is incidence angle. Model and measured data are specified in percentage.

Corrected refractive indices are presented in Fig. $\underline{6}$ by the gray curves. One can see that these curves are shifted from initial nominal refractive index wavelength dependencies in the direction of timecalibration refractive indices. These refractive indices were obtained on the basis of careful multiple characterizations of multilayer stacks in the course of long-term operation of the Helios deposition plant. This confirms that possible calibration inaccuracies are already eliminated for further considerations.
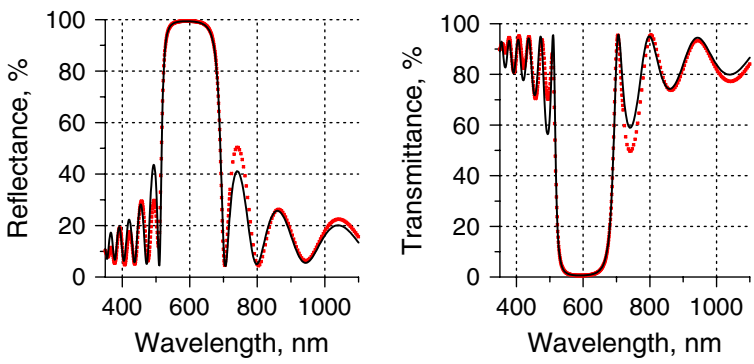

Fig. 8. (Color online) Fittings of measurement $R$ and $T$ data (crosses) by model reflectance and transmittance data (solid curves) obtained after the first step of the reverse-engineering procedure.

The achieved fittings of measured $R_{s}$ and $T_{s}$ taken at $7^{\circ}$ by corresponding model spectral characteristics are presented in Fig. 8 .

In Fig. 8, noticeable deviations of ripples of model spectral characteristics from ripples of measured characteristics are still observed. According to the analysis described in [5], these deviations can be connected with random errors in layer thicknesses $d_{1}, \ldots, d_{m}$. We consider relative errors in layer thicknesses and find these errors by minimizing the discrepancy function estimating a closeness between model and measured $R$ and $T$ data:

$$
\begin{aligned}
\mathrm{DF}^{2}= & \frac{1}{L} \sum_{j=1}^{L}\left\{\left[R \left(\tilde{n}_{H}\left(\lambda_{j}\right), \tilde{n}_{L}\left(\lambda_{j}\right),\right.\right.\right. \\
& \left.\left.\times\left(1+\delta_{1}\right) d_{1}, \ldots,\left(1+\delta_{m}\right) d_{m}, \lambda_{j}, \theta\right)-\hat{R}\left(\lambda_{j}, \theta\right)\right]^{2} \\
& +\left[T\left(\tilde{n}_{H}\left(\lambda_{j}\right), \tilde{n}_{L}\left(\lambda_{j}\right),\left(1+\delta_{1}\right) d_{1}, \ldots,\left(1+\delta_{m}\right) d_{m}, \lambda_{j}, \theta\right)\right. \\
& \left.\left.-\hat{T}\left(\lambda_{j}, \theta\right)\right]^{2}\right\} .
\end{aligned}
$$

In Eq. (3), $\delta_{1}, \ldots, \delta_{m}$ are relative errors in layer thicknesses, and $\tilde{n}_{H}=n_{H}+h_{H}$ and $\tilde{n}_{L}=n_{L}+h_{L}$ are corrected refractive indices found at the previous step of the reverse-engineering procedure.

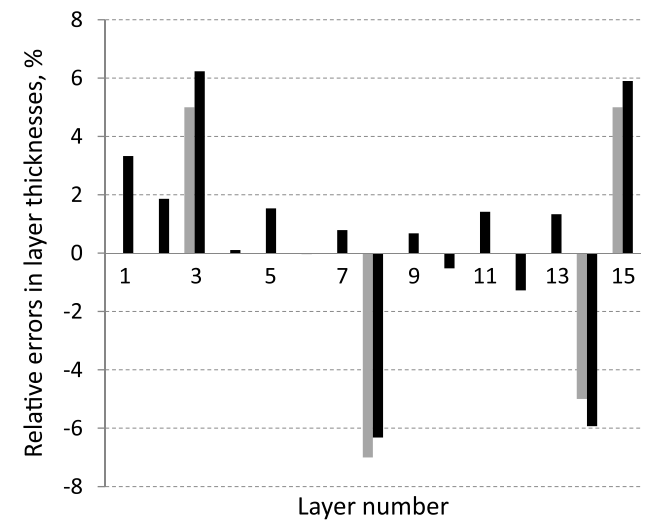

Fig. 9. Relative errors in thicknesses of 15-layer quarter-wave mirror determined from reflectance and transmittance data measured at $7^{\circ}$ incidence angle, $s$-polarization case (black bars) and planned errors in the thicknesses of third, eighth, 14th, and 15th layers (gray bars). 

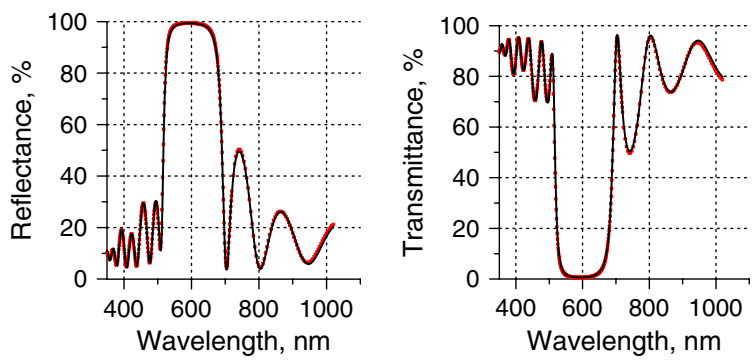

Fig. 10. (Color online) Fittings of measurement $R$ and $T$ data (crosses) by model reflectance and transmittance data (solid curves) achieved after the second step of reverse-engineering procedure.

Found relative errors in layer thicknesses are shown in Fig. $\underline{9}$ by the black bars. The obtained fittings of measured reflectance and transmittance data by corresponding model data are presented in Fig. 10. One can see in Fig. 9 that all intentionally made errors are reliably detected by the reverseengineering procedure. Deviations of found relative errors from the planned levels of errors (gray bars in Fig. 9) do not exceed estimated levels of errors associated with the time-monitoring procedure.

Analogous reverse-engineering procedures are performed with other combinations of analyzed measurement data. In all cases, we use experimental data in the spectral range from 330 to $1100 \mathrm{~nm}$. With growing incidence angle $R$ and $T$, spectral dependencies are shifted in the direction of the short wavelength range and, for this reason, there are fewer ripples of the spectral curves in the considered spectral range for high incidence angles. To provide more informative input data, we used combinations of obliqueincidence measurements for reverse-engineering procedures. Results of all reverse-engineering attempts are consistent. A typical example of the consistency of obtained results is presented in Fig. 11. Three sets of bars shown in this figure present relative errors in layer thicknesses of the examined mirror that are

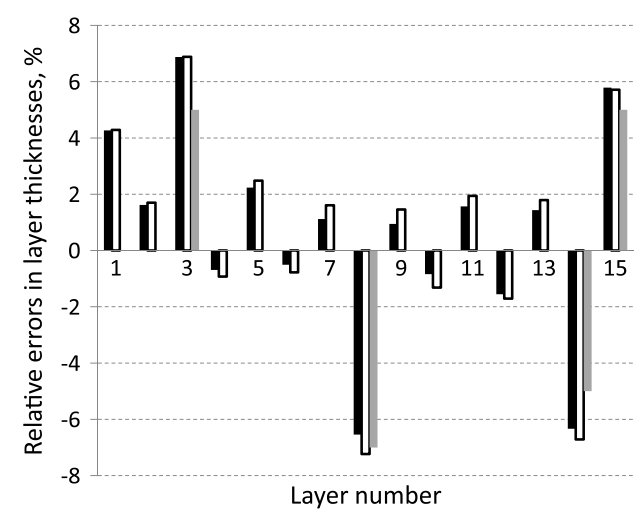

Fig. 11. Comparison of errors in layer thicknesses of 15-layer quarter-wave mirror found on the basis of reflectance and transmittance data taken at $7^{\circ}, 10^{\circ}, 20^{\circ}, 30^{\circ}$, and $40^{\circ}$, for the $s$ polarization case (black bars) and the $p$-polarization case (empty bars). Gray bars show planned errors in the thicknesses of the third, eighth, 14th, and 15th layers. found from three combinations of input measurement data: $T_{s}$ and $R_{s}$ data at $7^{\circ}$ incidence; $T_{s}$ and $R_{s}$ data at $7^{\circ}, 10^{\circ}, 20^{\circ}, 30^{\circ}$, and $40^{\circ}$ incidence; and $T_{p}$ and $R_{p}$ data at $7^{\circ}, 10^{\circ}, 20^{\circ}, 30^{\circ}$, and $40^{\circ}$ incidence.

One can see that, for all combinations of input data, intentional thickness errors are reliably detected. It also worth mentioning that all reverseengineering attempts detect a presence of the most noticeable unintentional error in the thickness of the first mirror layer. It is quite possible that this error is related to the mode of growth of the first layer at the substrate.

\section{Application of Multiangle Spectroscopy to Optical Characterization of Inhomogeneous E-Beam Evaporated Thin Films}

In this section, we consider single films of $\mathrm{SiO}_{2}$ and $\mathrm{HfO}_{2}$ and a multilayer system consisting of 12 alternate $\mathrm{HfO}_{2}$ and $\mathrm{SiO}_{2}$ quarter-wave layers. As in Section 3, we perform characterization of $\mathrm{SiO}_{2}$ and $\mathrm{HfO}_{2}$ films using separate oblique-incidence measurements for different polarization states. This allows us to check the consistency of the obtained results. The optical characterization procedure follows the methodology described in [4]. We use the Cauchy model to describe the dispersion behavior of $\mathrm{SiO}_{2}$ and $\mathrm{HfO}_{2}$ refractive indices. We assume a nonabsorbing model for $\mathrm{SiO}_{2}$ films and an exponential model [4] for the extinction coefficient of $\mathrm{HfO}_{2}$. Comparison of measured reflectance and transmittance data with corresponding spectral characteristics of the uncoated BK7 substrate indicate a presence of thin film bulk inhomogeneity in both films. For this reason, bulk inhomogeneity of $\mathrm{SiO}_{2}$ and $\mathrm{HfO}_{2}$ films is included in thin film models that we use in the course of the characterization process.

All characterization results that we obtain for $\mathrm{SiO}_{2}$ and $\mathrm{HfO}_{2}$ films are consistent. Variations in the obtained refractive index values do not exceed $0.2 \%$ for $\mathrm{SiO}_{2}$ film and $0.3 \%$ for $\mathrm{HfO}_{2}$ film; variations in layer thicknesses are less than $0.15 \%$ for $\mathrm{SiO}_{2}$ film, which is $503 \mathrm{~nm}$, and less than $0.3 \%$ for $\mathrm{HfO}_{2}$ film, which is $197 \mathrm{~nm}$ thick. The found degrees of bulk inhomogeneity are close to $-0.5 \%$ for $\mathrm{SiO}_{2}$ films and $-3.0 \%$ for $\mathrm{HfO}_{2}$ films. Extinction coefficient values of $\mathrm{HfO}_{2}$ are in the range of $0.2 \cdot 10^{-3}-0.3 \cdot 10^{-3}$ at the UV edge of $330 \mathrm{~nm}$, and are below $10^{-5}$ for wavelengths larger than $500 \mathrm{~nm}$.

Refractive index wavelength dependence of $\mathrm{SiO}_{2}$ is shown in Fig. 12 by the black solid curve. This dependence, as well as the $\mathrm{HfO}_{2}$ dependence below, is obtained on the basis of analysis of all available multiangle measurement data simultaneously, and again we call it a nominal refractive index. Two other curves in Fig. 12 show the reference $\mathrm{SiO}_{2}$ refractive index wavelength dependencies from [4] that were obtained for $\mathrm{SiO}_{2}$ films produced by magnetron sputtering and ion-beam sputtering. As one should expect, the refractive index of $\mathrm{SiO}_{2}$ film produced by e-beam evaporation is lower than the two other indices. This is in agreement with the fact that high 


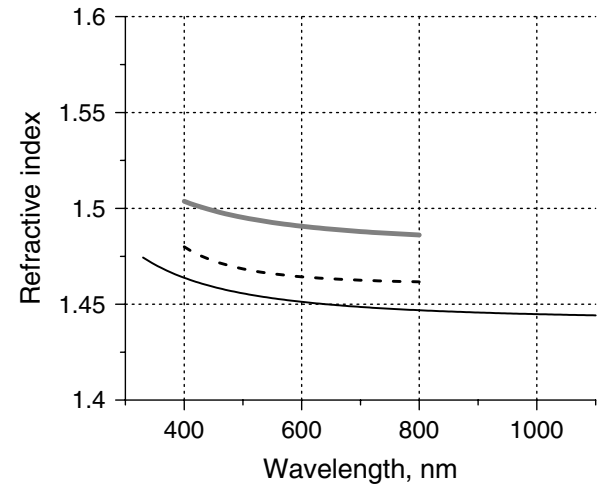

Fig. 12. Nominal refractive index wavelength dependence of ebeam evaporated $\mathrm{SiO}_{2}$ film (solid black curve), reference refractive index wavelength dependencies of $\mathrm{SiO}_{2}$ films produced by magnetron sputtering (dashed black curve), and ion-beam sputtering (gray curve).

energetic processes allow one to produce more dense films. It is also seen from Fig. 12 that a general pattern of the obtained wavelength dependence is in a good agreement with reference wavelength dependencies. As discussed in [4] in the case of amorphous dielectric films, differences in film densities shall reveal themselves mainly as shifts of refractive index wavelength dependencies up or down.

Figure 13 compares the found nominal refractive index of $\overline{\mathrm{HfO}}_{2}$ film with reference refractive index wavelength dependencies of $\mathrm{HfO}_{2}$ films from [4]. The reference films were produced by different $\overline{\mathrm{de}}$ position techniques: radio-frequency sputtering and ion-beam sputtering. One can clearly see that the dispersive behavior of the found $\mathrm{HfO}_{2}$ refractive index differs noticeably from the dispersive behavior of the reference $\mathrm{HfO}_{2}$ films. The found $n(\lambda)$ wavelength dependence is more steep than two other dependencies. This result is confirmed by the analysis of various separate oblique-incidence $R$ and $T$ measurement data.

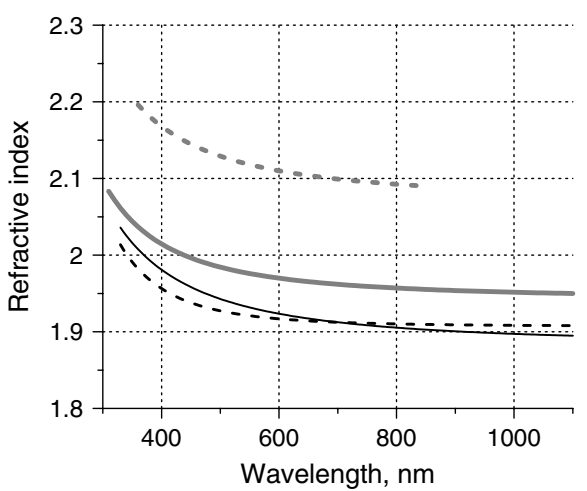

Fig. 13. Nominal refractive index wavelength dependence of ebeam evaporated $\mathrm{HfO}_{2}$ film (solid black curve), and reference refractive index wavelength dependencies of $\mathrm{HfO}_{2}$ films produced by radio frequency sputtering (gray curve) and ion-beam sputtering (dashed gray curve). The dashed black curve shows the refractive index of thin $\mathrm{HfO}_{2}$ film found from measurement data related to a 12-layer quarter-wave mirror (see the text for details).
It is obvious that the considered $\mathrm{HfO}_{2}$ film is less dense than two $\mathrm{HfO}_{2}$ films from [4]. For this reason, its refractive index is lower than two reference refractive indices. But there should also be another physical reason for the above-mentioned difference in steepness of the wavelength dependencies in Fig. 13. The most likely reason is a partial crystallization of the e-beam evaporated $\mathrm{HfO}_{2}$ films.

It has been already mentioned that the optical properties of thin film may be dependent on film thickness. This obviously relates to dielectric films with partial crystallization. Thin films in multilayer structures are usually several times thinner than thin films used for optical characterization. It is, therefore, possible that the optical parameters of e-beam evaporated $\mathrm{HfO}_{2}$ films in multilayer structures differ from those presented by the solid black curve in Fig. 13.

It is practically impossible to obtain optical parameters of films with thicknesses of a few tens of nanometers on the basis of their measured reflectance and transmittance. The dependencies of $R$ and $T$ in a spectral range typical for photometry do not have oscillating features and this dramatically degrades the accuracy of optical characterization. However, for optical characterization of dielectric films having thicknesses of a few tens of nanometers, another approach can be used. In [5], we proposed using reverse engineering of a quarter-wave mirror for the determination of refractive index wavelength dependencies of thin films forming this mirror.

Next we consider a 12-layer quarter-wave mirror with $\mathrm{HfO}_{2}$ and $\mathrm{SiO}_{2}$ layers. The central wavelength of this mirror is $410 \mathrm{~nm}$ and this means that the thicknesses of $\mathrm{HfO}_{2}$ and $\mathrm{SiO}_{2}$ layers are about 50 and $70 \mathrm{~nm}$, respectively. Both values are several times less than the thicknesses of $\mathrm{HfO}_{2}$ and $\mathrm{SiO}_{2}$ films used for optical characterization in the first part of this section.

To obtain convincing results, we again perform reverse engineering for different combinations of input measurement data and check the consistency of all obtained results. In the course of the reverseengineering procedures, we follow the same two-step algorithm that was proposed in [5]. At the first step, we refine the refractive indices of $\mathrm{HfO}_{2}$ and $\mathrm{SiO}_{2}$. We use the nominal refractive indices of single films depicted in Figs. 12 and $\underline{13}$ as initial dependencies. In the case of $\mathrm{SiO}_{2}$, only one parameter, a possible offset of the wavelength dependence found in the course of characterization of single layer sample, is determined. In the case of $\mathrm{HfO}_{2}$, we refine all three parameters of the Cauchy model of refractive index wavelength dependence. This is connected with the supposed difference in dispersive behavior of thick and thin $\mathrm{HfO}_{2}$ films. At the second step of the reverse-engineering procedure, we determine random errors in layer thicknesses.

Results of all reverse-engineering attempts are consistent. The offsets of $\mathrm{SiO}_{2}$ refractive indices determined in the course of reverse engineering are in 
the range from $1.5 \%$ to $1.7 \%$ with respect to the nominal refractive index of $\mathrm{SiO}_{2}$ found from characterization of the single $\mathrm{SiO}_{2}$ layer. A good agreement in refractive indices is observed in the case of $\mathrm{HfO}_{2}$ layers, as well. Variations of $\mathrm{HfO}_{2}$ refractive index values, found on the basis of separate obliqueincidence $R$ and $T$ measurements, do not exceed $0.5 \%$. Wavelength dependence of $\mathrm{HfO}_{2}$ refractive index, obtained as an average over all dependencies found on the basis of separate oblique-incidence measurements, is shown in Fig. 13 by the dashed black curve. In this figure, one can see that the found refractive index wavelength dependence of $\mathrm{HfO}_{2}$ film is now in agreement with reference wavelength dependencies from [4]. This agreement, obtained for small layer thicknesses in multilayer and the above-mentioned differences noticed for nominal refractive index of $\mathrm{HfO}_{2}$ having essentially larger single layer thickness, confirm the previously obtained conclusion that the crystalline state of $\mathrm{HfO}_{2}$ depends on the film thickness $[24,25]$. As shown in these references, thin films are basically amorphous while thicker films are partially crystalline, with larger crystalline fraction the thicker the film. Such behavior can explain the difference in refractive indices of our $\mathrm{HfO}_{2}$ films that are $197 \mathrm{~nm}$ thick in the case of a single layer and approximately $50 \mathrm{~nm}$ thick in the case of a multilayer structure.

\section{Conclusions}

In this paper, we studied the applicability of multiangle spectroscopy to optical characterization of thin films and reverse engineering of multilayers. A new advanced spectrophotometric accessory developed by Agilent Technologies supplied reflectance and transmittance data for multiple angle and $s$ - and $p$ polarization states. The accuracy of measurement data was verified and it was confirmed that all measurement data have high accuracy in a wide spectral range from the UV to the NIR up to the incidence angles of $40^{\circ}$.

Optical characterization of high-density $\mathrm{Ta}_{2} \mathrm{O}_{5}$ and $\mathrm{SiO}_{2}$ films produced by magnetron sputtering demonstrated excellent consistency of thin film optical parameters obtained using separate obliqueincidence spectroscopic data. A specially prepared quarter-wave mirror with intentional errors in several $\mathrm{Ta}_{2} \mathrm{O}_{5}$ and $\mathrm{SiO}_{2}$ layers was used for checking the reliability of the reverse-engineering procedures performed using various combinations of input measurement data. The estimated levels of random errors in high and low index layers were several times less than intentional thickness errors. In all experiments with various combinations of input measurement data, the intentional thickness errors were reliably detected. Consistency among the results of various experiments was also demonstrated.

We applied multiangle spectral photometric data for the determination of optical parameters of ebeam evaporated $\mathrm{HfO}_{2}$ and $\mathrm{SiO}_{2}$ films with various thicknesses. In the case of thin films, a special ap- proach based on reverse engineering of a specially prepared multilayer mirror was used for this purpose. It was found that optical properties of e-beam evaporated $\mathrm{HfO}_{2}$ films are essentially dependent on film thickness. Reliability of the obtained results is confirmed by experiments with various combinations of input measurement data.

Multiangle spectral photometry provides researchers with more experimental information than conventional spectroscopy. Our study demonstrates that the currently available multiangle spectrophotometer accessory provides experimental information that has sufficient accuracy for solving various characterization and reverse-engineering problems. Comparative analysis of various combinations of input multiangle spectroscopic data provides selfverification of obtained characterization and reverse-engineering results. We believe that multiangle spectral photometry has good prospects for the analysis of optical coatings that are supposed to be used at oblique light incidence or at diverged light illumination. Further experiments are planned in this direction.

This work was supported by the German Research Foundation Cluster of Excellence, Munich-Centre for Advanced Photonics (http:www.munich-photonics .de), by the Russian Fund of Basic Research, project 10-07-00480-a, and by the National Foundation of Science, Higher Education and Technological Development of Croatia.

\section{References}

1. A. Duparre and D. Ristau, "2004 Topical Meeting on Optical Interference Coatings: measurement problem," in Optical Interference Coatings, OSA Technical Digest Series (Optical Society of America, 2004), paper WD1.

2. A. Duparre and D. Ristau, "Optical Interference Coatings 2007 measurement problem," Appl. Opt. 47, C179-C184 (2008).

3. A. Duparre and D. Ristau, "2010 OSA Topical Meeting on Optical Interference Coatings: measurement problem," in Optical Interference Coatings, OSA Technical Digest (Optical Society of America, 2010), paper ThC1.

4. A. Tikhonravov, M. Trubetskov, T. Amotchkina, G. DeBell, V. Pervak, A. K. Sytchkova, M. Grilli, and D. Ristau, "Optical parameters of oxide films typically used in optical coating production," Appl. Opt. 50, C75-C85 (2011).

5. A. Tikhonravov, M. Trubetskov, T. Amotchkina, A. Tikhonravov, D. Ristau, and S. Gunster, "Reliable determination of wavelength dependence of thin film refractive index," Proc. SPIE 5188, 331-342 (2003).

6. A. V. Tikhonravov and M. K. Trubetskov, OptiLayer thin film software, http://www.optilayer.com.

7. A. V. Tikhonravov and M. K. Trubetskov, "On-line characterization and reoptimization of optical coatings," Proc. SPIE 5250, 406-413 (2004).

8. J. Woollam, "Ellipsometry, variable angle spectroscopic," in Wiley Encyclopedia of Electrical and Electronics Engineering, J. Webster, ed. (Wiley, 2000). Supplement 1.

9. A. V. Tikhonravov, "Design and reverse engineering of optical coatings," in Optical Interference Coatings (Optical Society of America, 1998) Vol. 9, pp. 300-301.

10. R. Andrade, E. Birgin, I. Chambouleyron, J. Martinez, and S. Ventura, "Estimation of the thickness and the optical parameters of several stacked thin films using optimization," Appl. Opt. 47, 5208-5220 (2008). 
11. K. Lamprecht, W. Papousek, and G. Leising, "Problem of ambiguity in the determination of optical constants of thin absorbing films from spectroscopic reflectance and transmittance measurements," Appl. Opt. 36, 6364-6371 (1997).

12. D. Poelman and P. Smet, "Methods for the determination of the optical constants of thin films from single transmission measurements: a critical review," J. Phys. D 36, 1850-1857 (2003).

13. S. Nevas, F. Manoocheri, E. Ikonen, A. V. Tikhonravov, M. A. Kokarev, and M. K. Trubetskov, "Optical metrology of thin films using high-accuracy spectro-photometric measurements with oblique angles of incidence," Proc. SPIE 5250, 234-242 (2004).

14. A. Lamminpää, S. Nevas, F. Manoocheri, and E. Ikonen, "Characterization of thin films based on reflectance and transmittance measurements at oblique angles of incidence," Appl. Opt. 45, 1392-1396 (2006).

15. W. V. Goodell, J. K. Coulter, and P. B. Johnson, "Optical constants of Inconel alloy films," J. Opt. Soc. Am. 63, 185-188 (1973).

16. W. V. Goodell, J. K. Coulter, and P. B. Johnson, "Derivation of optical constants of metals from thin-film measurements at oblique incidence," Appl. Opt. 11, 643-651 (1972).

17. T. Kihara and K. Yokomori, "Simultaneous measurement of the refractive index and thickness of thin films by $s$-polarized reflectances," Appl. Opt. 31, 4482-4487 (1992).
18. V. Pervak, M. Trubetskov, and A. Tikhonravov, "Robust synthesis of dispersive mirrors," Opt. Express 19, 2371-2380 (2011).

19. V. Pervak, C. Teisset, A. Sugita, S. Naumov, F. Krausz, and A. Apolonski, "High-dispersive mirrors for femtosecond lasers," Opt. Express 16, 10220-10233 (2008).

20. P. A. van Nijnatten, "An automated directional reflectancetransmittance analyser for coating analysis," Thin Solid Films 442, 74-79 (2003).

21. A. Tikhonravov, M. Trubetskov, and G. DeBell, "On the accuracy of optical thin film parameter determination based on spectrophotometric data," Proc. SPIE 5188, 190-199 (2003).

22. H. Kaiser, "Film deposition materials," in Optical Interference Coatings, N. Kaiser and H. K. Pulker, eds. (Springer-Verlag, 2003), pp. 131-153.

23. V. Pervak, "Recent developments and new ideas in the field of dispersive multilayer optics," Appl. Opt. 50, C55-C61 (2011).

24. M. Modreanu, J. Sancho-Parramon, D. O’Connell, J. Justice, O. Durand, and B. Servet, "Solid phase crystallisation of $\mathrm{HfO}_{2}$ thin films," Mater. Sci. Eng. B 118, 127-131 (2005).

25. M. Modreanu, J. Sancho-Parramon, O. Durand, B. Servet, M. Stchakovsky, C. Eypert, C. Naudin, A. Knowles, and F. Bridou, "Investigation of thermal annealing effects on microstructural and optical properties of $\mathrm{HfO}_{2}$ thin films," Appl. Surface Sci. 253, 328-334 (2006). 